\title{
CATECHOLAMINE RESPONSES IN PARAPLEGIA
}

\author{
By D. B. Frewin, M.D., B.S., M. Levitt, Ph.D., S. J. Myers, M.D., \\ C. C. Co, M.D. and J. A. Downey, M.D., D.Phil.(Oxon) \\ Department of Rehabilitation Medicine, College of Physicians and Surgeons, \\ Columbia University, New York and the New York State Psychiatric Institute
}

\section{INTRODUCTION}

THE activity of sympathetic nerves and the adrenal medulla may be estimated by measuring urinary catecholamines. Von Euler, Hellner-Bjorkman and Orwen (I955), reported that in normal subjects the excretion of noradrenaline and adrenaline was higher during the day than at night. Naftchi, et al. (I972), have shown that chronic paraplegics have an augmented urinary output of catecholamine metabolites. In the present study, the diurnal urinary excretion of the adrenaline and noradrenaline were examined in one paraplegic subject during the subacute phase of his spinal injury. We also compared his values with those obtained from a group of five chronic paraplegics. The effect was studied of a cardiovascular stress (tilt) on his heart rate, mean arterial pressure and plasma Dopamine- $\beta$-Hydroxylase activity.

\section{MATERIALS AND METHODS}

Subjects. I. E. B. a 42-year-old white male, was involved in a motor-cycle accident on 18 September 1972 and suffered an injury to his spine at the level of the fifth, sixth and seventh thoracic vertebrae. Surgical exploration performed at another hospital, confirmed the presence of a fracture through the laminae of $\mathrm{T}_{5}, 6$, and 7 and the body of $\mathrm{T}_{7}$. Also, complete rupture of the posterior longitudinal ligament and extrusion of the T6/7 intervertebral disc. There was tearing of the dura at the T6/7 level and extrusion of the spinal cord through the hiatus. The cord appeared to be completely crushed at this site and the emerging nerve roots lacerated. In addition to injuries of the spinal cord, the patient suffered a laceration of the $(R)$ lobe of the liver, trauma to the gall bladder and common bile duct, dislocation of the $(\mathrm{R})$ hip and fracture of several ribs. He was transferred to the Rehabilitation Medicine Unit of the Columbia-Presbyterian Medical Centre three weeks later for the management of his spinal injury.

At the time of admission the patient had a complete loss of motor and sensory function below the level of the umbilicus. He was conscious, cooperative and alert with a supine blood pressure of $130 / 70 \mathrm{~mm}$. $\mathrm{Hg}$ and a pulse rate of 70 beats per minute. His heart and lungs were normal. A drainage tube was present in the common bile duct. The past medical history included a doudenal ulcer ro years previously from which he was completely symptom free at the time of admission to hospital.

Four days after being admitted to Rehabilitation Medicine, the patient passed a large, tarry stool, following which his blood pressure fell to $60 / 40 \mathrm{~mm}$. $\mathrm{Hg}$ and his pulse rate increased to I 30 beats per minute. Fresh blood was aspirated from the stomach. The patient was transferred to the Medical Intensive Care Unit and treated there until the bleeding abated. When recovery from the above 
episode was complete, a T-tube cholangiogram was performed. Following this the patient became febrile up to $104^{\circ} \mathrm{F}$. with chills. A rigor and blood cultures revealed a growth of $E$. coli which responded to appropriate antibiotic therapy. The remainder of the patient's stay in hospital was uneventful and he was discharged on 2I December 1972 to the care of an out-patient clinic. At that time he was independent, in self-care in a wheelchair, living at home with his wife and family.

2. Five chronic ( 6 months-I 5 years) clinically complete, traumatic paraplegic subjects aged $18-55$ (mean 33.4 years) acted as patient controls. The levels of their lesion ranged from Ti to L2 (Table I). All medications that could interfere with sympathetic nerve activity were discontinued at least two weeks prior to the test.

3. Four normal controls participated in the second part of the study. They were aged $24-42$ (mean age $29 \cdot 5$ years).

\section{TABLE I}

Clinical data for the chronic paraplegic (Control) subjects

\begin{tabular}{|c|c|c|c|c|c|c|c|}
\hline \multirow{2}{*}{ Subject } & \multirow{2}{*}{ Sex } & \multirow{2}{*}{$\begin{array}{c}\text { Age } \\
\text { (years) }\end{array}$} & \multirow{2}{*}{$\begin{array}{l}\text { Level of } \\
\text { lesion }\end{array}$} & \multirow{2}{*}{$\begin{array}{l}\text { Duration of } \\
\text { paralysis } \\
\text { (months) }\end{array}$} & \multicolumn{3}{|c|}{$\begin{array}{l}\text { Urinary catecholamines } \\
\quad(\mu \mathrm{g} . / 24 \text { hours })\end{array}$} \\
\hline & & & & & $\mathbf{N}$ & A & Total \\
\hline R. M. & $M$ & I 8 & $T_{I}$ & 9 & $48 \cdot 82$ & $7 \cdot 72$ & $56 \cdot 54$ \\
\hline J. S. & M & 42 & $\mathrm{~T}_{\mathrm{I}}$ & I 2 & $32 \cdot 54$ & 5.93 & 38.47 \\
\hline P.S. & $\mathrm{M}$ & I9 & T6 & 7 & 34.55 & $7 \cdot 74$ & $42 \cdot 29$ \\
\hline J. M. & $\mathrm{M}$ & 33 & $\mathrm{~T}_{\mathrm{I} 2 / \mathrm{LI}}$ & I86 & 43.05 & $9 \cdot 23$ & $52 \cdot 28$ \\
\hline R. C. & $\mathrm{M}$ & 55 & $\mathrm{~L} 2$ & 6 & $93 \cdot 70$ & I9.24 & I 12.94 \\
\hline
\end{tabular}

Experimental Procedure. All patients had catheter drainage at the time of the study. Urine samples were collected in six-hourly aliquots in $500 \mathrm{ml}$. polyethylene bottles containing $5 \mathrm{ml}$. of $6 \mathrm{~N} \mathrm{HCl}$. The times of collection were from I200-I800, I800-2400, 2400-0600 and 0600-I200 hours respectively. The samples were frozen until assayed for adrenaline and noradrenaline, using a modification of the method of Hanlon (Hanlon, 1970).

Patient E. B. was studied on four separate occasions, 3, 6, I0 and I4 weeks after sustaining his spinal injury, the last study being the day prior to his discharge from hospital. The collections were made when his cardiovascular signs were stable and he was not suffering from intercurrent illnesses or complications.

Tilt Studies. Patient E. B., two chronic paraplegics and four normal controls participated in this part of the study. After 30 minutes lying flat they were rapidly tilted to the $45^{\circ}$ foot-down position. Blood pressure and heart rate were measured before, during and after the tilt. The subjects were returned to the horizontal after Io minutes or sooner if they experienced symptoms of impending syncope. E. B., who was studied six weeks after sustaining his injury, was only able to tolerate nine minutes of tilting, while J. M. and R. C., who were both 'tilt tolerant' did not have any symptoms during the test.

Blood samples for the measurement of plasma Dopamine- $\beta$-Hydroxylase activity $(\mathrm{DBH})$ were obtained via an indwelling cannula in an ante-cubital vein 
after 20 and 30 minutes' rest in the supine position, on the 5 th and Ioth minute of the tilt (nine minutes in the case of E. B.) and half an hour after the subject was returned to the horizontal position. The samples were collected in heparinised tubes, placed on ice, centrifuged and the plasma frozen at $-65^{\circ} \mathrm{C}$. until assayed. Their DBH activity was estimated using a modification of the method described by Nagatsu and Udenfriend (1972). Duplicate aliquots of each plasma sample were assayed and the mean value used in the calculation. The DBH activity was expressed as $\mu \mathrm{mol} / \mathrm{minute} /$ litre.

\section{Urinary Catecholamines $(\mu \mathrm{g} / 24 \mathrm{Hrs})$}

$180-$
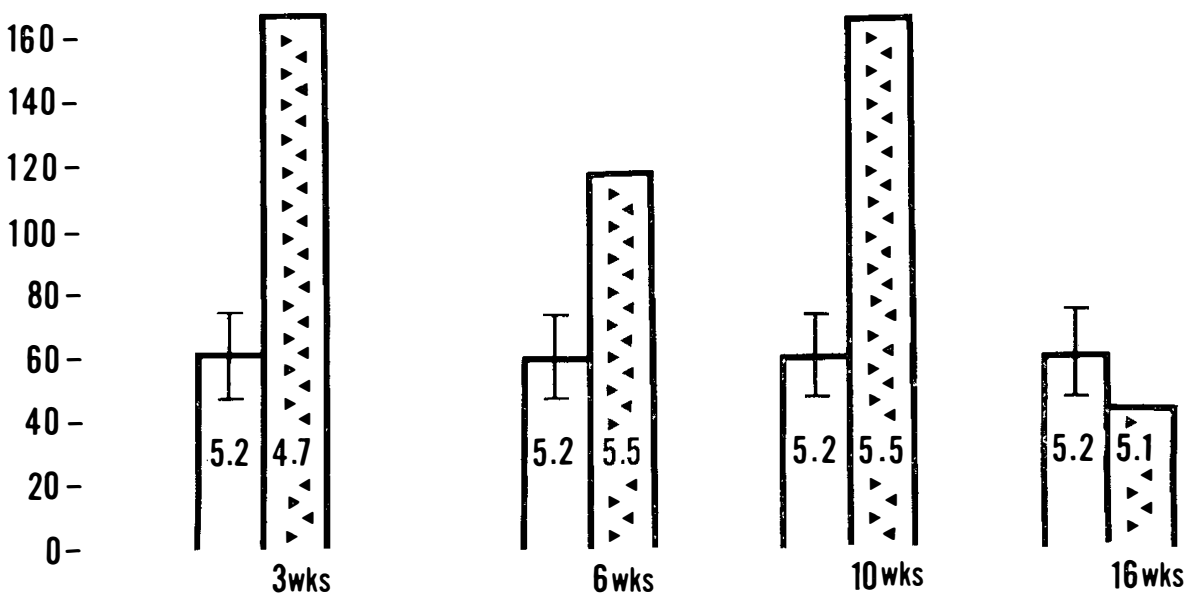

FIG. I

The shaded bars represent E. B.'s daily catecholamine excretion 3, 6, I0 and 16 weeks after his spinal injury. The unshaded bars represent the mean daily catecholamine excretion for the five chronic paraplegic patients. The figures within the bars are the noradrenaline: adrenaline ratios in the sample and the vertical lines, the standard errors of the mean.

\section{RESULTS}

Figure I illustrates E. B.'s total urinary catecholamine excretion on the four separate occasions. The first time being three weeks after his injury, and the last, one day prior to his discharge from hospital. Compared with the values of the control paraplegics, his catecholamine excretion was markedly elevated during the first ro weeks of the injury. However, at the time of his leaving hospital, when postural conditioning was complete, his urinary catecholamine levels were in the same range as the chronic paraplegics. Despite the marked difference in E. B.'s total urinary catecholamine values, the ratios of adrenaline: noradrenaline in the samples were similar to the controls (fig. I). The values for the daily excretion of adrenaline and noradrenaline in the chronic paraplegics are shown in Table I. There did not appear to be any relationship between the level of the lesion and the magnitude 
$60-$

\section{Urinary Noradrenaline Excretion}
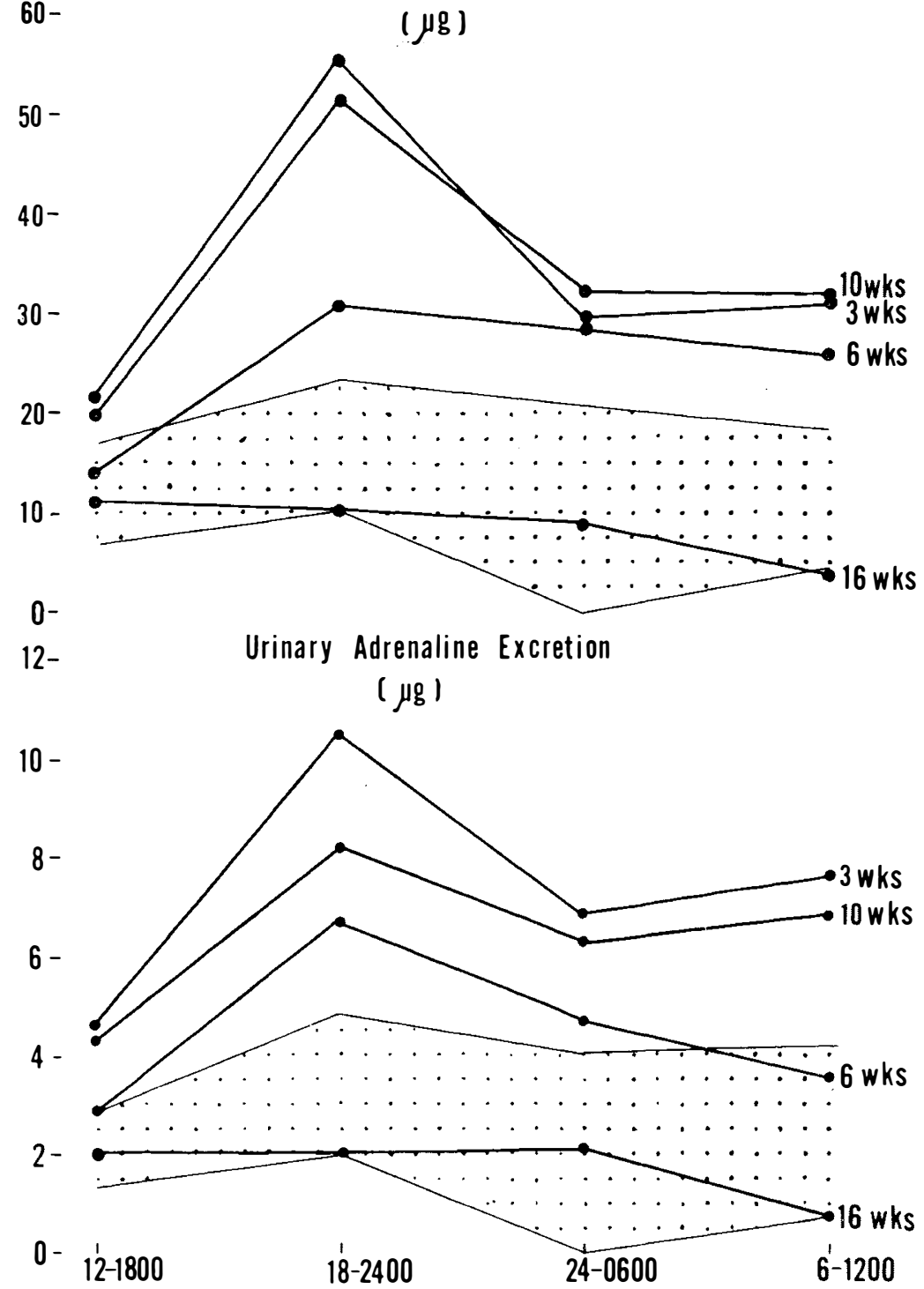

HOURS

FIG. 2

The values for E. B.'s urinary noradrenaline (upper frame) and adrenaline (lower frame) excretion during the four standard collection periods, 3, 6, Io and I 6 weeks after spinal injury. The hatched areas in both frames include one standard deviation about the mean value for the chronic paraplegic controls. 
of the catecholamine excretion. However, the highest values were obtained for R. C. who had an injury to his lumbar cord.

Figure 2 illustrates the diurnal variation in E. B.'s urinary adrenaline and noradrenaline excretion during the 14 weeks after spinal injury. In most of the studies, the highest value for adrenaline and noradrenaline occurred in the sample obtained between 1800 and 2400 hours. The pattern of excretion of the two catecholamines was essentially the same, but the levels of adrenaline were approximately one-fifth of those of noradrenaline. As E. B.'s recovery progressed, the excretion of catecholamines became more uniform and similar to the chronic paraplegic controls.

\section{Heart Rate (b.p.m.)}

\section{DBH Activity} ( $\mu \mathrm{mol} / \mathrm{min} /$ litre)
Mean Arterial Pressure (mm $\mathrm{Hg}$ )

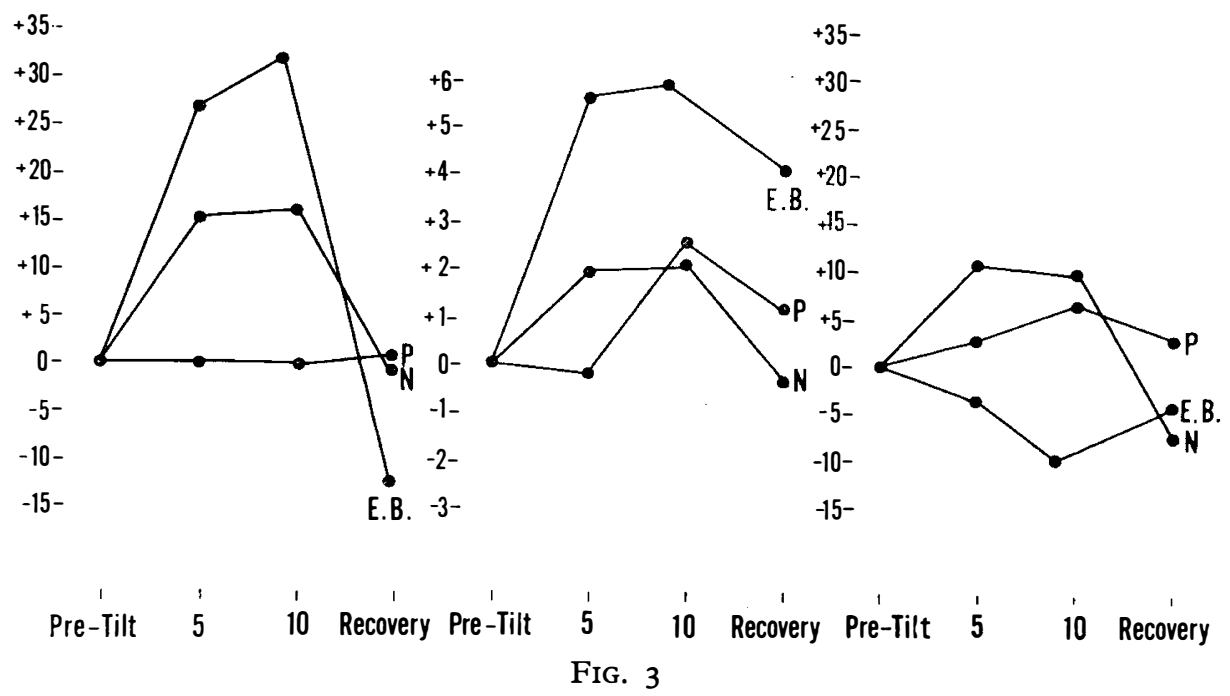

The changes in heart rate, plasma DBH activity and mean arterial pressure which occurred during the $45^{\circ}$ foot-down tilt. E. B.'s responses are shown at the 5th and 9th minute, as he was unable to tolerate the standard ro minute period. The mean responses for the normals $(\mathrm{N})$ and chronic paraplegics $(\mathrm{P})$ are also shown. The mean of the two control readings has been designated as 'Zero-Pre-Tilt' and the changes in the three parameters determined relative to this value; increases are denoted as $(+)$ and decreases as $(-)$. Mean arterial pressure is derived from the sum of diastolic pressure with one-third the pulse pressure.

Figure 3 shows the changes in heart rate, plasma DBH activity and mean arterial pressure. These were recorded during the foot-down tilt to $45^{\circ}$ in patient E. B., the normal controls and the two chronic paraplegic subjects. During the tilt the normal subjects had I5-I7 b.p.m. increase in heart rate, a small rise in plasma $\mathrm{DBH}$ activity and a rise in mean arterial pressure. In contrast, the two paraplegics had no increase in heart rate. There was a rise in $\mathrm{DBH}$ activity which was of the same magnitude as the normals and a small rise in mean arterial pressure. E. B., however, responded with a very striking increase in heart rate and plasma $\mathrm{DBH}$ activity, but despite these responses felt faint during the 9th minute of tilting and had to be returned to the horizontal position. 


\section{DISCUSSION}

The results of this study indicate that in the subacute phase of E. B.'s spinal injury, the urinary excretion of catecholamines was high in comparison with the levels of these amines excreted by chronic paraplegics. As recovery progressed, his urinary adrenaline and noradrenaline levels fell to the range of the paraplegic controls. Despite the elevation in total catecholamine excretion, the ratio of noradrenaline : adrenaline remained constant throughout this period, and was similar to that obtained in a normal man (von Euler et al., I955, \& Karki, I956).

Tilting the subjects to the $45^{\circ}$ foot-down position produced cardiovascular changes. The four normal subjects had a significant increase in heart rate and mean arterial pressure, and a small rise in plasma $\mathrm{DBH}$ activity. The latter enzyme is localised in sympathetic nerves and catalyses the terminal step in the biosynthesis of noradrenaline. It is released together with this transmitter when the sympathetic nerves are stimulated (Gewirtz \& Kopin, 1970). Wooten and Cardon (I973), studied the effect of tilting on plasma DBH activity and found the response of the enzyme to be variable. In four of six normal subjects they studied, there was a small increase in enzyme activity while in two, the level of activity decreased. Our two chronic paraplegics who were both 'tilt tolerant', did not have any change in heart rate during the tilt but had an increase in plasma DBH activity comparable with the normals. Both patients had lesions of the lumbar cord and it is not clear why they did not get a cardioaccelerator response. However, the fact that adequate vascular compensation did occur was borne out by the rise in mean arterial pressure during the tilting. Our findings substantiate those of Guttmann et al. (1963), who showed that the incidence of fainting in subjects with lesions below T6 level was only slightly higher than in normal subjects undergoing similar tests. The increase in plasma DBH activity in the patients is in keeping with Guttmann et al.'s observations that paraplegics have a rise of plasma catecholamines during tilting.

Patient E. B. had a very significant increase in heart rate and a rise in DBH activity which was approximately three times that of the normal and chronic paraplegic subjects. However, despite these changes his blood pressure fell, making him dizzy and faint on the 9th minute of the tilt. In paraplegic subjects, vasomotor adjustments to tilting are lost in the denervated area (Bidart \& Maury, 1973) and it is conceivable that this factor contributed to E. B.'s fall in blood pressure. However, the results must be viewed in the light of many complications the patient suffered from during the first few weeks following his injury. These could have affected his ability to maintain cardiovascular homeostasis in the erect position. The large cardioaccelerator response he had, probably represented an over-compensation for the failure in vasomotor adjustment. This phenomenon has been noted previously in patients with dysautonomia (Frank et al., 1972). Our studies on E. B. were performed six weeks after his injury whilst he was receiving postural conditioning. We were unable to study him again in this manner, but it would seem likely that the clinical improvement he demonstrated was associated with a return in his ability to compensate by increasing his vascular resistance.

The diurnal catecholamine excretion was also interesting. The highest excretion in the chronic paraplegic patients was between I800 and 2400 hours. E. B. had a similar, but greater excretion, especially earlier in his illness. Previous studies on normal subjects have shown that the mean level of urinary noradrenaline and adrenaline excretion is highest during the day and reduced at night (Karki, 
1956). As E. B. 'recovered', there was a gradual 'flattening out' of the diurnal level of catecholamine excretion, and a return of his values to the range occupied by the chronic paraplegic controls. We have not compared the total daily catecholamine excretion of the paraplegics with the figures given for normal subjects by von Euler et al. (1955) and Karki (1956), as there was a difference in the methodology used in these studies. However, the values obtained by us for the patients appear to be considerably higher than those reported by them, for the normal subjects.

The overall catecholamine response in the paraplegic would therefore appear to depend on (I) the duration of the injury and (2) postural conditioning. The mechanisms that operate are of an essentially compensatory nature and are an attempt at producing cardiovascular homeostasis. However, if the autonomic deficit is large, compensation is inadequate and results in postural hypotension and syncope. This is disabling to the patients and therapeutic methods must be devised to compensate for the deficits.

Acknowledgement. The assistance of Dr. A. Karchmar and Mr. W. K. Luke is gratefully acknowledged. This work was supported in part by HSMHA grant No. 21586 to M. L.

\section{REFERENCES}

Bidart, Y. \& MAURY, M. (I973). Paraplegia, II, I.

Frank, H. J., Frewin, D. B., Robinson, S. M. \& Wise, P. H. (1972). Aust. N.Z. f. Med. I, I.

GewirTz, G. P. \& Kopin, I. J. (I970). Nature (Lond.) 227, 406.

Guttmann, L., Munro, A. F., Robinson, R. \& Walsh, J. J. (1963). Paraplegia I, 4.

HANLON, J. F. (1970). Analytical Biochem. 34, 568.

Karki, N. T. (I956). Acta. Physiol. Scand. 39, Suppl. I32, I.

Naftchi, N. E., Lowman, E. W., Sell, G. H. \& Rusk, H. A. (1972). Arch. Phys. Med. 53, 357.

Nagatsu, T. \& Udenfriend, S. (1972). Clin. Chem. I 8, 980.

Von Euler, U. S., Hellner-Bjorkman, S. \& Orwen, I. (1955). Acta Physiol. Scand. 33, Suppl. I I8, IO.

Wooten, F. G. \& Cardon, P. V. (1973). Arch. Neurol. 28, I03. 